\title{
Une homologie frappante entre le CFTR et les annexines
}

\author{
Hugues Chap, Josette Fauvel, Ama Gassama-Diagne, \\ Jeannie Ragab-Thomas, Marie-Françoise Simon
}

L'isolement récent du gène de la mucoviscidose a permis de prédire la séquence de la protéine dont le déficit fonctionnel serait responsable de la maladie héréditaire autosomique récessive la plus fréquente ([1] et $\mathrm{m} / \mathrm{s}$ $n^{\circ}$ 8, vol. 5, p. 589). Le CFTR (cystic fibrosis transmembrane conductance regulator) apparaît ainsi comme un nouveau membre d'une super-famille de protéines membranaires impliquées dans le transport, dépendant de l'ATP, de diverses substances à l'extérieur des cellules. Cependant, la relation entre le CFTR et les altérations fonctionnelles du canal à chlore $\left(\mathrm{Cl}^{-}\right)$, caractéristiques de la mucoviscidose sont encore mal comprises, la nature du composé expulsé de la cellule par le CFTR n'étant pas élucidée.

L'analyse génétique a attiré l'attention sur l'une des deux régions potentielles de liaison de l'ATP située à proximité de l'extrémité N-terminale du CFTR (NBF1, nucleotide binding fold). En effet, celle-ci renferme en position 508 un résidu de phénylalanine dont la délétion est retrouvée chez $68 \%$ des patients ou des transmetteurs [2]. De plus, l'anomalie fonctionnelle du canal $\mathrm{Cl}$ - peut être corrigée par transfert et expression de l'ADNc codant pour le CFTR dans des cellules de malades.porteurs de

\section{ADRESSE}

H. Chap : professeur à la faculté de médecine de Toulouse-Purpan. J. Fauvel : chef de travaux à la faculté de médecine de Toulouse-Purpan. A. Gassama-Diagne : chercheur post-doctoral. J. Ragab-Thomas : maître de conférences à la faculté de médecine de Toulouse-Purpan. M.-F. Simon : char gée de recherche à l'Inserm. Inserm U. 326, Phospholipides membranaires, signalisation cellulaire et lipoprotéines, hôpi- la délétion de la phénylalanine 508 $[3,4]$.

Les annexines, appelées aussi lipocortines $\left(\mathrm{m} / \mathrm{s} n^{\circ} 5\right.$, vol. 3, p. 282), constituent une famille de protéines se liant aux membranes en présence de calcium, par une double interaction avec les phospholipides anioniques et le cytosquelette, mais dont le rôle est encore mal défini [5]. En plus des phosphorylations spécifiques de certaines annexines par des protéines tyrosine kinases sur leur extrémité $\mathrm{N}$ terminale, cinq d'entre elles possèdent un site de phosphorylation par la protéine kinase $\mathrm{C}$, localisé sur le premier des quatre domaines répétés caractéristiques de ces protéines. Par simple examen visuel, nous avons noté une homologie relativement frappante entre ce site de phosphorylation et le segment peptidique qui précède la phénylalanine $508 \mathrm{du}$ CFTR. Cela a été confirmé par la recherche systématique de séquences homologues au segment 500-529 du CFTR, qui peut être aligné avec la séquence de 8 annexines différentes (figure 1), le degré d'homologie étant compris entre $47 \%$ (annexine III) et $37 \%$ (annexines I et VIII).

Une telle homologie est certainement trop limitée en longueur pour proposer une relation évolutive claire entre les deux types de protéines. Cependant, cette observation nous suggère les remarques suivantes : (1) il existe un degré frappant de conservation de certains résidus, en particulier la phénylalanine 508 ; (2) l'homologie observée est spécifique et ne concerne pas le NBF2 ou les autres protéines apparentées au CFTR ; (3) le CFTR et les annexines ont des localisations cellulaires semblables (par exemple, dans l'espace sous-membranaire de cellules épithéliales, comme l'annexine II).

Bien que nous ne puissions pas exclure une similitude purement fortuite, nous suggérons que la séquence homologue pourrait participer à des interactions communes des annexines et du CFTR avec des composés cellulaires qui restent à identifier. De telles interactions protéiques se dévoilent de plus en plus dans le domaine de la transduction des signaux cellulaires [6] ou interviennent dans l'association de l'annexine II avec un polypeptide de $11 \mathrm{kDa}$ apparenté aux protéines S100 [7]. De plus, il a été suggéré que le CFTR pourrait être impliqué dans l'extrusion de substances lipidiques telles que les leucotriènes [8]. Des études ultérieures devraient permettre de tester l'hypothèse d'une liaison éventuelle du domaine NBF1 du CFTR avec certains lipides, ce qui correspond à la caractéristique essentielle des annexines. Enfin, un très récent travail rapportait une modulation par l'annexine VI de l'activité d'un canal calcique du réticulum sarcoplasmique [9], ce qui constituerait une similitude fonctionnelle avec le CFTR, censé intervenir dans la régulation de la conductance aux ions $\mathrm{Cl}-\mathrm{de}$ la membrane de diverses cellules épithéliales

\section{TIRÉS A PART}

H. Chap. 


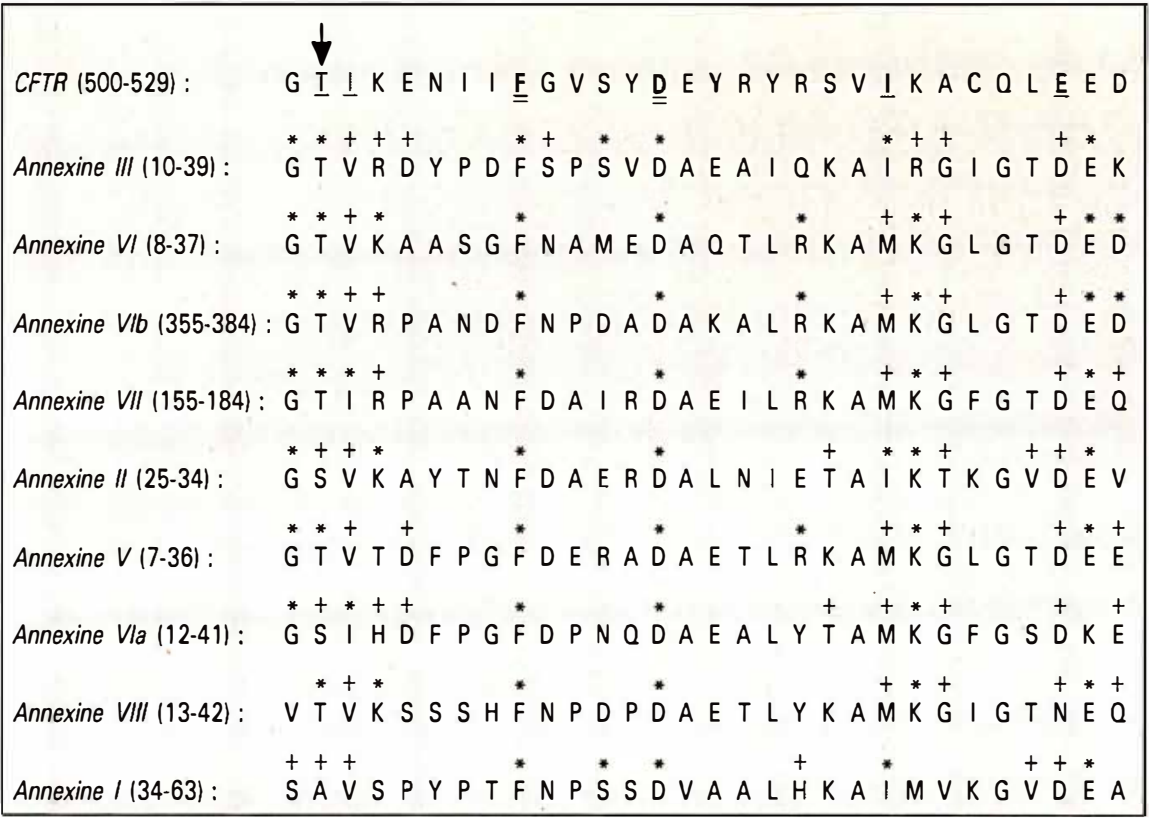

Figure 1. Alignement de la séquence 500-529 du CFTR avec les séquences correspondantes des annexines. La comparaison a été effectuée au sein de la banque SWISS-PROT à l'aide du programme SCANSIM. Quatre annexines (II, IV, VIb, VII) sont significativement semblables à la sous-séquence du CFTR selon le test du programme PCOMPARE (score > 5). Les annexines sont classées de haut en bas par ordre d'homologie décroissante avec le CFTR. Les identités sont indiquées par *, les substitutions conservatrices par + ldéfinies par les groupes suivants: $S, T, A, G, P ; N, D, E, Q ; R, K, H ; M, l$, $L, V ; F, Y, W)$. Les résidus doublement soulignés correspondent à ceux intégralement conservés; ceux simplement soulignés indiquent des substitutions entièrement conservatrices. Les termes Vla et VIb indiquent les deux parties de I'annexine VI (domaines répétés $1-4$ et $5-8$, respectivement). La flèche signale le site potentiel de phosphorylation par la protéine kinase $C$.

\section{RÉFÉRENCES}

1. Riordan JR, Rommens JM, Kerem BS, et al. Identification of the cystic fibrosis gene : cloning and characterization of complementary DNA. Science $1989 ; 245$ : 1066-72.

2. Kerem BS, Rommens JM, Buchanan JA, et al. Identification of the cystic fibrosis gene : genetic analysis. Science 1989 ; $245: 1073-80$.

3. Rich DP, Anderson MP, Gregory RJ, et al. Expression of cystic fibrosis transmembrane conductance regulator corrects defective chloride channel regulation in cystic fibrosis airway epithelial cells. Nature 1990 ; $347: 358-63$.

4. Drumm ML, Pope HA, Cliff WH, et al. Correction of the cystic fibrosis defect in vitro by retrovirus-mediated gene transfer. Cell $1990 ; 62: 1227-33$

5. Klee $\mathrm{CB}$. $\mathrm{Ca}^{2+}+$-dependent phospholipid- (and membrane-) binding proteins. Biochemistry $1990 ; 27$ : 6646-53.

6. Kaplan DR, Morrison DK, Wong G, McCormick F, Williams LT. PDGF $\beta$ receptor stimulates tyrosine phosphorylation of GAP and association of GAP with a signaling complex. Cell $1990 ; 61: 125-33$. 7. Johnsson N, Marriott G, Weber K. p36, the major cytoplasmic substrate of src tyrosine protein kinase, binds to its 11 regulatory subunit via a short amino-terminal amphipatic helix. EMBO J 1988; 7 : 2435-42.

8. Ringe D, Petsko GA. Cystic fibrosis. A transport problem? Nature 1990 ; 346 : 312-3.

9. Diaz-Munoz M, Hamilton SL, Kaetzel MA, Hazarika P, Dedman JR. Modulation of $\mathrm{Ca}^{2}+$ release channel activity from sarcoplasmic reticulum by annexin VI (67 kDa calcimedin). J Biol Chem 1990 ; 265: 15894-9.

\section{Summary}

A striking homology between CFTR and annexins

A significant homology (47-37\%) has been observed between subsequence 500-529 of CFTR (cystic fibrosis transmembrane conductance regulator) and 8 different annexins. This segment includes the functionnally very critical phenylalanine 508 residue, which is fully conserved among annexins. This suggests some possible common interactions of CFTR and annexins with other yet unidentified cell components present in or close to the submembraneous space. 